\title{
Factors determining early adherence to a lung cancer screening protocol
}

\author{
U. Montes*,", L.M. Seijo*, A. Campo*, A.B. Alcaide*, G. Bastarrikađ and J.J. Zulueta*
}

ABSTRACT: Lung cancer screening using computed tomography (CT) is effective in detecting early stage disease. However, concerns regarding adherence have been raised.

The current authors conducted a retrospective observational study of 641 asymptomatic smokers enrolled in a lung cancer screening programme between 2000 and 2003. Adherent subjects were compared with nonadherent subjects with regard to lung function, sex, age, motivation for enrolment, smoking status, distance to the referral centre, family history of lung cancer, asbestos exposure, education, the presence and type of nodule(s) seen on initial CT, and exposure to a nursing intervention designed to improve adherence.

Overall, early adherence to the study protocol was $65 \%$. Multivariate analysis confirmed the importance of sex, proximity to the referral centre, the presence of noncalcified nodules, and the nursing intervention as factors conditioning adherence to the study protocol. Patients encouraged to participate in the study were more adherent, as were former smokers. Sex interactions were observed in multivariate analysis. The nursing intervention was significant for females, while abnormal lung function improved male adherence.

Adherence to lung cancer screening is particularly good among females and subjects living near the referral centre. The present study suggests the need to develop new strategies, especially those targeting males and subjects with low risk perception, in order to improve adherence.

KEYWORDS: Adherence, computed tomography, lung cancer, lung cancer screening

ung cancer is the worldwide leader of cancer deaths [1, 2]. In Europe alone, 400,000 new cases of lung cancer are diagnosed each year [3]. Since most lung cancers are diagnosed at a late stage [4], lung cancer survival remains poor, not exceeding $15 \%$ at 5 yrs [5]. Unfortunately, lung cancer screening employing chest radiography and sputum cytology has been disappointing despite detecting a greater number of early stage tumours. Routine lung cancer screening is currently not recommended.

Recent advances in medical imaging technology have prompted re-evaluation of lung cancer screening using low-dose computed tomography (CT). Several studies, including the International Early Lung Cancer Action Program (I-ELCAP), are currently under way to determine whether lung cancer screening using CT is capable of detecting early-stage lung cancer and improving survival. Recently published data regarding I-ELCAP results have shown an $88 \% 10$-yr survival in patients with stage I lung cancer identified during screening [6]. Such outcomes demonstrate that lung cancer screening using CT can detect curable disease. Since adherence to cancer screening varies, concerns about adherence to the screening protocol have been raised.

The current retrospective study seeks to investigate patient characteristics and other factors conditioning adherence to the I-ELCAP study in Navarra, Spain.

\section{MATERIALS AND METHODS}

A retrospective study was conducted of 641 atrisk subjects participating in the University of Navarra lung cancer screening programme, a member of I-ELCAP, between 2000 and 2003.

\section{Design of the lung cancer screening programme}

The I-ELCAP study enrolled smokers or former smokers aged $\geqslant 40$ yrs who had smoked for $\geqslant 10$ pack-yrs. All subjects are informed of the programme's content, including timing of CT scanning. Once informed consent was obtained, the subject completed an epidemiological questionnaire from which much of the data for the present study were obtained.
AFFILIATIONS

*Depts of Pulmonary Medicine,

"Radiology, Clínica Universitaria, and

\#Center for Applied Medical

Investigation, University of Navarra,

Pamplona, Spain.

\section{CORRESPONDENCE}

L.M. Seijo

Pulmonary Medicine

Clinical University

University of Navarra

Avda. Pio XII

36

31008 Pamplona

Spain

Fax: 34948296500

E-mail: Imseijo@unav.es

Received:

November 032006

Accepted after revision:

May 222007

STATEMENT OF INTEREST

None declared.

European Respiratory Journal

Print ISSN 0903-1936

Online ISSN 1399-3003 


\section{I-ELCAP protocol}

The institutional bioethics committee (Navarra, Spain) approved the study protocol prior to implementation. Decision-making followed the standard pre-approved protocol, details of which are beyond the scope of the present study and can be viewed on the I-ELCAP website [7].

\section{Adherence criteria}

Early adherent subjects were defined as those who returned for a second CT scan within 18 months of the initial study. Nonadherent subjects were those who never returned following the initial scan or took $>18$ months to do so.

\section{Factors and patient characteristics studied with respect to early adherence}

Subject characteristics were analysed in both the adherent and nonadherent groups with regard to: prevalence of chronic obstructive pulmonary disease (COPD), sex, age, motivation for enrolment, smoking status, education, family history of lung cancer, proximity to the referral centre, asbestos exposure, the presence and type of nodule(s) on initial CT scan, perception of risk, and exposure to a nursing intervention designed to improve adherence.

With regard to patient motivation, two groups were identified: 1) patients whose enrolment in the study was based on a personal initiative; and 2) patients who enrolled following family or medical advice.

\section{Nursing intervention}

A nursing intervention protocol, designed to promote adherence, was established at the University of Navarra, Pamplona, Spain from January 2003. A dedicated full-time nurse tracked adherence to staff recommendations regarding repeat CT scanning. Written and telephone reminders were sent to all participants in order to maximise adherence. At least one annual written reminder was sent to all subjects from January 2003. Every subject was telephoned at least once. Subjects with abnormal scans were singled out, and repeatedly telephoned in order to avoid drop out among high-risk participants. With regard to adherence, the group of subjects enrolled in the current study before and after the full-time nurse began tracking adherence, mailing reminders and calling subjects with abnormal scans to improve adherence, were compared.

\section{Statistical analysis}

Univariate and multivariate analysis was conducted.

\section{RESULTS}

Overall, early adherence to the I-ELCAP study at the authors' centre was $65 \%$. Factors conditioning adherence were as follows.

\section{Distance to the referral centre}

In total, $80 \%$ of subjects living within $200 \mathrm{~km}$ (125 miles) of the referral centre returned for at least one repeat scan within 18 months of the initial study compared with $58 \%$ of subjects living beyond that range $(p<0.001)$. The distance to the referral centre remained significant on multivariate analysis (odds ratio (OR) 2.55, 95\% confidence interval (CI) 1.65-3.95).

\section{Sex}

Females proved to be more adherent to the study protocol than males $(p<0.001)$. Adherence among female participants reached $75 \%$ compared with $57 \%$ among males. Multivariate analysis confirmed the significance of sex (OR 2.07, 95\% CI 1.41-3.04). Furthermore, multivariate analysis revealed an important interaction between sex and other factors conditioning adherence. The nursing intervention (OR 10.01, 95\% CI 4.90-20.45), presence of noncalcified nodules (OR 3.35, 95\% CI 1.47-7.61) and distance to the referral centre (OR 2.55, 95\% CI 1.12-4.99) conditioned female adherence. Male adherence was dependent on abnormal lung function (OR 2.09, 95\% CI 1.193.67), distance to the referral centre (OR 2.33, 95\% CI 1.33-4.07), age (OR 1.07, 95\% CI 1.03-1.10), smoking status (OR 1.86, 95\% CI 1.03-3.36), motivation (OR 1.69, 95\% CI 1.01-2.82) and the presence of noncalcified nodules (OR 2.42, 95\% CI 1.43-4.10).

\section{Type of nodule}

In total, $74 \%$ of subjects with at least one noncalcified pulmonary nodule returned for a second scan compared with $61 \%$ of subjects without noncalcified pulmonary nodules $(\mathrm{p}<0.01)$. This held true for males and females in multivariate analysis (OR 2.75, 95\% CI 1.77-4.29).

\section{Nursing intervention}

The nursing intervention improved adherence on univariate $(\mathrm{p}<0.001)$ and multivariate analysis (OR 3.12, 95\% CI 2.10$4.84)$, but it did not significantly improve adherence among males (fig. 1).

\section{Motivation for participation in the study and smoking status} Motivation was a significant factor conditioning adherence on multivariate analysis (OR 1.54, 95\% CI 1.03-2.30). Subjects encouraged to participate in lung cancer screening by their family or physician were more adherent. Former smokers were also more adherent than current smokers (OR 2.00, 95\% CI 1.21-3.28).

\section{Risk perception}

It has been shown previously at the 13th International Conference on Screening for Lung Cancer that subjects with a greater risk perception, as well as those most afraid, of developing lung cancer are more adherent to lung cancer screening $(\mathrm{p}<0.05)$.

\section{Factors not conditioning adherence}

Cumulative pack-years of smoking, asbestos exposure, age, education level (high school or less versus university), and family history of lung cancer had no effect on adherence. Adherent and nonadherent subjects accounted for 26.5 and 25.6 pack-yr smoking histories, respectively (fig. 2 and table 1).

\section{Self-reported reasons for nonadherence}

Self-reported reasons for nonadherence among 52 subjects contacted by telephone included: oversight or lack of time ( $44 \%)$; distance to the referral centre (21\%); a "benign initial $\mathrm{CT}^{\prime \prime}(14 \%)$; and cost (12\%). 

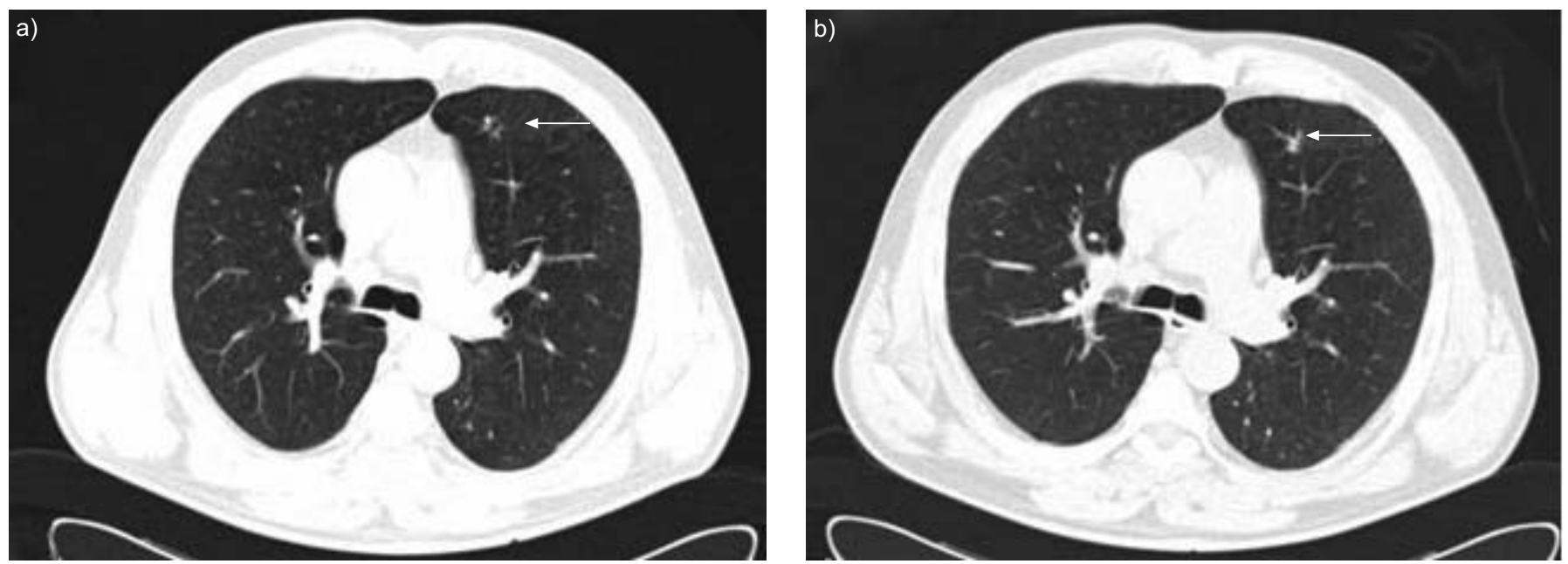

FIGURE 1. Computed tomography scans from a patient requiring four telephone reminders following the initial scan (a). The follow-up study (b) showed obvious growth of a nodule in the left upper lobe (arrows). The patient underwent resection of a stage T1NOMO mucinous adenocarcinoma.

\section{DISCUSSION}

The chief objective of lung cancer screening is to reduce mortality among subjects at risk, while minimising undue harm or anxiety attributable to false-positive test results. Adherence rates will inevitably determine success of established screening programmes.

\section{Defining adherence}

Studies of cancer screening are limited by the lack of a uniform definition of adherence. KHANNA et al. [8] offer a broad definition focusing on follow-up. They defined nonadherence as a failure to undergo follow-up or treatment within a specified period of time following abnormal findings. The current authors chose to focus on early adherence to follow-up, and established relatively flexible criteria (i.e. 18 months). Given the natural history of lung cancer, any follow-up study performed beyond 30 months probably reflects prevalence

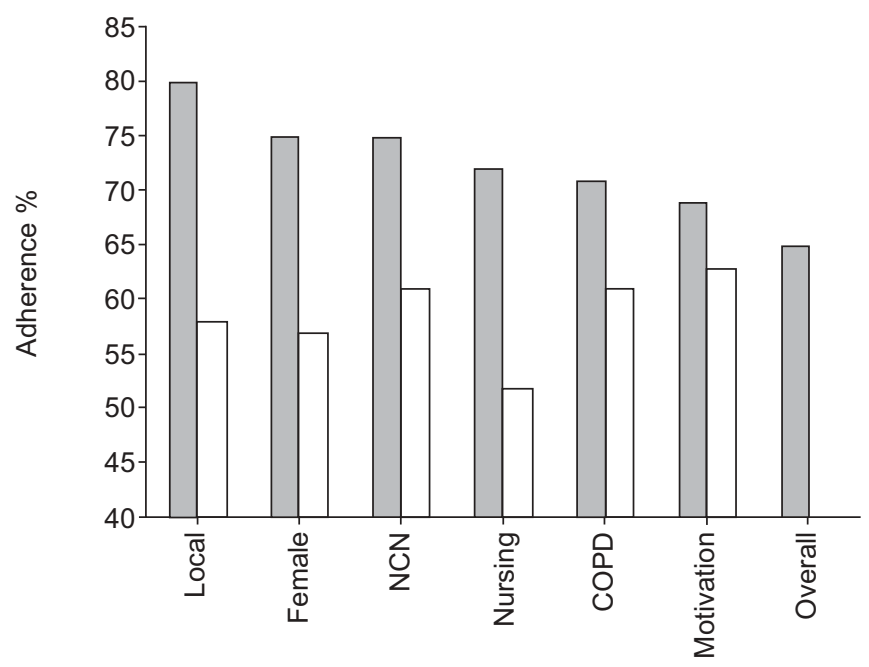

Factors

FIGURE 2. Histograms showing the factors which condition adherence. NCN: noncalcified nodules; COPD: chronic obstructive pulmonary disease. 1 : yes; $\square$ : no. rather than incidence of disease [9]. Very few patients returned for a second scan between 18-30 months. They were excluded from data analysis since classifying them as adherent or nonadherent would have been arbitrary.

\section{Adherence to established cancer screening programmes}

Nonadherence to screening programmes precludes successful screening initiatives. In a retrospective study of 647 patients referred for colonoscopy, adherence did not exceed $50 \%$ [10, 11]. Other groups have found similar or even lower rates of adherence for screening programmes employing sigmoidoscopy or faecal occult blood testing [12, 13]. Adherence to

\begin{tabular}{|c|c|c|}
\hline Factors & Odds ratio & p-value \\
\hline \multicolumn{3}{|l|}{ Univariate analysis } \\
\hline Distance & & $<0.001$ \\
\hline Females & & $<0.001$ \\
\hline Noncalcified nodule on baseline CT & & $<0.001$ \\
\hline Nursing Intervention & & $<0.001$ \\
\hline COPD & & $<0.001$ \\
\hline Heightened risk perception & & $<0.05$ \\
\hline Fear of lung cancer & & $<0.05$ \\
\hline \multicolumn{3}{|l|}{ Multivariate analysis } \\
\hline Distance & 2.33 & \\
\hline Females & 2.07 & \\
\hline Noncalcified nodule on baseline CT & 3.35 & \\
\hline \multicolumn{3}{|l|}{ Nursing intervention } \\
\hline Females & 10.01 & \\
\hline Motivation for screening & 1.69 & \\
\hline \multicolumn{3}{|l|}{ Abnormal lung function } \\
\hline Males & 2.09 & \\
\hline Age & 1.07 & \\
\hline Smoking status & 1.86 & \\
\hline
\end{tabular}

CT: computed tomography; COPD: chronic obstructive pulmonary disease. 
cervical cancer screening is variable. There have been reports of $80 \%$ adherence rates to planned Pap smears [14]. Conversely, breast cancer screening is much more successful. Adherence often exceeds $90 \%$ [14, 15]. For example, the breast cancer screening programme in Navarra accomplished $88 \%$ adherence between 1990 and 2002 [16].

Overall adherence to the present study compares favourably with established colon and cervical cancer screening programmes, but is still disappointing when compared with breast cancer screening. The University of Navarra faces unique limitations, including an abundance of referred patients who must travel several hundred miles to the referral centre. Interestingly, $80 \%$ of the study subjects living close to the referral centre were adherent. If sex is taken into account, with males being far less adherent than females, perhaps adherence is not far off from the enviable breast cancer screening statistics from the present authors' own community.

\section{Factors conditioning adherence to cancer screening}

DENBERG et. al. [10] have identified several factors that condition adherence to colonoscopy, including: age, female sex and type of insurance. Nonadherent patients contacted by telephone reported low risk perception, fear of endoscopy and its potential complications (including pain), cost and logistics as reasons for nonadherence. The current study population reported similar reasons for nonadherence. TAYLOR et al. [17] described factors conditioning adherence to lung cancer screening, including false-positive test results, female sex and education level. Interestingly, sex had the opposite effect in the present study. This finding may be relevant to the future of screening, since smoking is at an all-time high among females [18]. Female smokers are at increased risk of developing lung cancer compared with males [18, 19], and lung cancer deaths already exceed breast cancer deaths in the USA [3]. Low adherence rates among males are a matter of concern, especially in countries such as Spain, where males still account for the majority of new lung cancer cases. The present findings suggest that future strategies dedicated to improving adherence may need to be sex-specific.

False-positive results are also troublesome, as they seem to condition adherence to a number of screening programmes including colon, prostate and ovarian cancer. A false-positive result may diminish confidence in the validity of future test results while denying the patient the desired reassurance. According to the Health Belief Model [20] there is a clear association between how a patient is informed of abnormal screening test results, and faith in the value of cancer screening in general. Paradoxically, false-positive results may increase short-term or early adherence while diminishing long-term adherence [17]. This may be relevant to lung cancer screening since the presence of noncalcified nodules (most of which are benign) is a significant factor conditioning early adherence to the current study, probably by increasing anxiety or personal cancer risk perception. It should be considered whether the abundance of benign pulmonary nodules (present in $>25 \%$ of all scans) may also prove counterproductive in terms of securing adherence to lung cancer screening in the long run. In this regard, FORD et al. [21] found that adherence to lung cancer screening was lower among patients with false-positive chest radiograph results, especially current smokers. Only future studies will clarify the true impact of false positives, and especially that of noncalcified benign pulmonary nodules on adherence rates to lung cancer screening using low dose CT.

Some of the factors conditioning adherence are of greater concern than others for the future of lung cancer screening. For example, distance to the referral centre does not represent a significant problem in Europe or the USA where CT technology is widely available. However, it may render lung cancer screening initiatives using spiral CT in third world countries ineffective. This is of great concern as lung cancer is quickly spreading throughout Africa and Asia [22-24]. Cost is yet another issue. If lung cancer screening becomes a reality who will cover the expense of testing and follow-up?

The success of a nursing intervention designed to improve adherence to cancer screening comes as no surprise, since several studies have found similar results [25-30]. LANTZ et al. [27] and RIMMER [31] have reported that simple mailed or telephone reminders can achieve a four-fold increase in adherence to breast and cervical cancer screening programmes. Similarly, other studies have shown that phoned advice and reminders increase adherence to follow-up among patients with abnormal screening test results $[8,15,32]$. Why males do not respond to telephone reminders in the current study as well as females do remains a mystery.

As patients with COPD have a greater risk of developing lung cancer [33], it is reassuring to find that males with abnormal lung function are more adherent to screening. At the authors' center alone, $68 \%$ of patients with screening-detected lung cancer had obstruction on spirometry. The reason why these individuals are more adherent may be related to increased anxiety or awareness of their risk, prevalence of chronic respiratory symptoms, or perhaps an abundance of abnormal scans (i.e. emphysema). JONES et al. [34] have reported that patients with chronic symptoms are more likely to consider the potential consequences of their illness, which in turn conditions greater compliance. Why females with abnormal lung function are not necessarily more adherent is also puzzling.

It has been previously shown that subjects with a heightened risk perception are more adherent. This is also true of breast cancer screening programmes [35], but has not been conclusively demonstrated in other types of cancers [36]. In fact, studies of cervical cancer screening have shown that fear of the diagnosis may have the opposite effect [37], limiting adherence, while subjects with low-risk perception of colon cancer tend to be less adherent, especially those who are asymptomatic [10] or have not received appropriate counselling [38]. Similar conclusions can be drawn from the Model of Planned Behaviour or the attitude, social influence and self-efficacy (ASE) model, which predict that patient behaviour varies as a consequence of attitudes, motivation to comply and social influence [19]. In the current study, subjects who underwent screening as a result of family insistence or professional counselling were found to be more adherent on multivariate analysis.

It was surprising to find that most nonadherent subjects contacted by telephone were well aware of the reasons for their failure to follow-up. Similar results have been reported in cervical and colorectal cancer screening $[8,37]$. In a study by FORD et al. [21] on lung cancer screening, nonadherent subjects 
reported lack of interest $(58 \%)$ or time $(27 \%)$ as common reasons for their failure to comply with screening, while distance to the referral centre $(5 \%)$, family affairs $(5 \%)$ or personal illness $(5 \%)$ were also mentioned.

\section{Study limitations}

A retrospective design is an obvious limitation in the current study, since data was collected from a questionnaire that was not designed to obtain information regarding adherence as an end-point. Changes in subject attitudes toward screening as a direct result of participation in the study, e.g. heightened anxiety related to abnormal CT scan findings, could not be measured. In addition, the questionnaire did not ask patients about household income, yet it was found that cost is one of the self-reported reasons for nonadherence. The true impact of nursing intervention is uncertain since the two groups were compared at different time periods, i.e. before and after 2003 when the nursing protocol was implemented. The present authors tried contacting a representative cohort of nonadherent subjects; however, any such initiative has obvious pitfalls. The unique limitations posed by the authors' institution, a tertiary referral centre, have already been commented upon, including distance and cost. As the authors' centre tends to attract higher income private patients who can afford to travel and the expense of screening, the true impact of cost on nonadherence might have been underestimated in the present study. Other limitations inherent to I-ELCAP include uncertainties about long-term follow-up (i.e. when should annual CT screening be terminated) and the abundance of benign pulmonary nodules, which might condition adherence in the long run.

\section{Conclusions}

Adherence to lung cancer screening using low-dose computed tomography remains a concern. Males and low risk perception subjects are particularly challenging, while cost, distance to the referral centre and other factors conditioning adherence pose additional challenges to widespread screening. Strategies to improve adherence, such as nursing intervention, have proven successful but may need to be sex-specific. Follow-up rates still need to be improved, perhaps, as some studies suggest, by improving patient feedback or active workshop participation $[31,39]$. Improving adherence is not synonymous with reducing unnecessary anxiety; counselling strategies designed to improve the former without exacerbating the latter need to be developed. Misconceptions regarding screening also need to be limited to ensure screened subjects understand that a new cancer which is not yet visible might be identified within 12 months, in order to avoid generating a false sense of security, perhaps by improving patient education regarding lung cancer screening.

\section{REFERENCES}

1 Jemal A, Murray T, Samuels A, Ghafoor A, Ward E, Thun MJ. Cancer statistics, 2003. CA Cancer J Clin 2003; 53: 5-26.

2 Franco J, Perez-Hoyos S, Plaza P. Changes in lung-cancer mortality trends in Spain. Int J Cancer 2002; 97: 102-105.

3 Boyle P, Dresler C. Preventing the lung cancer epidemic. Ann Oncol 2005; 16: 1565-1566.
4 Batura-Gabryel H, Foremska-Iciek J. Lung cancer in the elderly-increasing epidemiological problem of 21st century. Rocz Akad Med Bialymst 2005; 50: Suppl. 1, 152-155.

5 Mountain CF. Revisions in the International System for Staging Lung Cancer. Chest 1997; 111: 1710-1717.

6 Henschke CI, Yankelevitz DF, Libby DM, Pasmantier MW, Smith JP, Miettinen OS. Survival of patients with stage I lung cancer detected on CT screening. N Engl J Med 2006; 355: 1763-1771.

7 International Early Lung Cancer Action Program I-ELCAP. www.ielcap.org/members.htm Date last accessed: July 2007. Date last updated: July 2007.

8 Khanna N, Phillips MD. Adherence to care plan in women with abnormal Papanicolaou smears: a review of barriers and interventions. J Am Board Fam Pract 2001; 14: 123-130.

9 Bastarrika G, Garcia-Velloso MJ, Lozano MD, et al. Early lung cancer detection using spiral computed tomography and positron emission tomography. Am J Respir Crit Care Med 2005; 171: 1378-1383.

10 Denberg TD, Melhado TV, Coombes JM, et al. Predictors of nonadherence to screening colonoscopy. J Gen Intern Med 2005; 20: 989-995.

11 Detsky AS. Screening for colon cancer-can we afford colonoscopy? N Engl J Med 2001; 345: 607-608.

12 Larsen IK, Grotmol T, Almendingen K, Hoff G. Lifestyle characteristics among participants in a Norwegian colorectal cancer screening trial. Eur J Cancer Prev 2006; 15: 10-19.

13 Vernon SW. Participation in colorectal cancer screening: a review. J Natl Cancer Inst 1997; 89: 1406-1422.

14 Ruchlin HS. Prevalence and correlates of breast and cervical cancer screening among older women. Obstet Gynecol 1997; 90: 16-21.

15 Michielutte R, Sharp PC, Foley KL, et al. Intervention to increase screening mammography among women 65 and older. Health Educ Res 2005; 20: 149-162.

16 Ascunce N, Barcos A, Ederra M, et al. [Breast cancer screening program. Results of the process and impact indicators (1990-2002).] An Sist Sanit Navar 2004; 27: 319-333.

17 Taylor KL, Shelby R, Gelmann E, McGuire C. Quality of life and trial adherence among participants in the prostate, lung, colorectal, and ovarian cancer screening trial. J Natl Cancer Inst 2004; 96: 1083-1094.

18 Pauk N, Kubik A, Zatloukal P, Krepela E. Lung cancer in women. Lung Cancer 2005; 48: 1-9.

19 Henschke CI, Miettinen OS. Women's susceptibility to tobacco carcinogens. Lung Cancer 2004; 43: 1-5.

20 Becker MH, Maiman LA. Sociobehavioral determinants of compliance with health and medical care recommendations. Med Care 1975; 13: 10-24.

21 Ford ME, Havstad SL, Flickinger L, Johnson CC. Examining the effects of false positive lung cancer screening results on subsequent lung cancer screening adherence. Cancer Epidemiol Biomarkers Prev 2003; 12: 28-33.

22 Kaneko S, Ishikawa KB, Yoshimi I, et al. Projection of lung cancer mortality in Japan. Cancer Sci 2003; 94: 919-923.

23 Jee SH, Kim IS, Suh I, Shin D, Appel LJ. Projected mortality from lung cancer in South Korea, 1980-2004. Int J Epidemiol 1998; 27: 365-369. 
24 Sitas F, Urban M, Bradshaw D, Kielkowski D, Bah S, Peto R. Tobacco attributable deaths in South Africa. Tob Control 2004; 13: 396-399.

25 Duan N, Fox SA, Derose KP, Carson S. Maintaining mammography adherence through telephone counseling in a church-based trial. Am J Public Health 2000; 90: 1468-1471.

26 Cargill VA, Conti M, Neuhauser D, McClish D. Improving the effectiveness of screening for colorectal cancer by involving nurse clinicians. Med Care 1991; 29: 1-5.

27 Lantz PM, Stencil D, Lippert MT, Beversdorf S, Jaros L, Remington PL. Breast and cervical cancer screening in a low-income managed care sample: the efficacy of physician letters and phone calls. Am J Public Health 1995; 85: 834-836.

28 Bobo JK, Shapiro JA, Schulman J, Wolters CL. On-schedule mammography rescreening in the National Breast and Cervical Cancer Early Detection Program. Cancer Epidemiol Biomarkers Prev 2004; 13: 620-630.

29 Lacey L, Whitfield J, DeWhite W, et al. Referral adherence in an inner city breast and cervical cancer screening program. Cancer 1993; 72: 950-955.

30 Ansell D, Lacey L, Whitman S, Chen E, Phillips C. A nursedelivered intervention to reduce barriers to breast and cervical cancer screening in Chicago inner city clinics. Public Health Rep 1994; 109: 104-111.

31 Rimmer BK. Adherence to cancer screening. Cancer Control 1995; 2: 510-517.
32 Melnikow J, Chan BK, Stewart GK. Do follow-up recommendations for abnormal Papanicolaou smears influence patient adherence? Arch Fam Med 1999; 8: 510-514.

33 Papi A, Casoni G, Caramori G, et al. COPD increases the risk of squamous histological subtype in smokers who develop non-small cell lung carcinoma. Thorax 2004; 59: 679-681.

34 Jones SL, Jones PK, Katz J. Compliance in acute and chronic patients receiving a health belief model intervention in the emergency department. Soc Sci Med 1991; 32: 1183-1189.

35 Katapodi MC, Lee KA, Facione NC, Dodd MJ. Predictors of perceived breast cancer risk and the relation between perceived risk and breast cancer screening: a meta-analytic review. Prev Med 2004; 38: 388-402.

36 Hiatt RA, Klabunde C, Breen N, Swan J, Ballard-Barbash R. Cancer screening practices from National Health Interview Surveys: past, present, and future. J Natl Cancer Inst 2002; 94: 1837-1846.

37 Lerman C, Hanjani P, Caputo C, et al. Telephone counseling improves adherence to colposcopy among lower-income minority women. J Clin Oncol 1992; 10: 330-333.

38 Petersen GM. Barriers to preventive intervention. Gastroenterol Clin North Am 2002; 31: 1061-1068, viii.

39 Stone EG, Morton SC, Hulscher ME, et al. Interventions that increase use of adult immunization and cancer screening services: a meta-analysis. Ann Intern Med 2002; 136: 641-651. 